\title{
Validación del coeficiente de rugosidad de Manning en un canal a flujo libre bajo condición de flujo uniforme
}

\section{Validating the Manning's Roughness Coefficient in a Free-flowing Channel under Steady Flow Conditions}

\author{
Recibido: 06 de septiembre de 2020. \\ Recibido en revisión: 16 de octubre de 2020. Aceptado:05 de noviembre de 2020 \\ DOl: https://doi.org/10.33132/27114260.1935 \\ Nicolás M. Solano ${ }^{3}$, Mónica V. Sandoval ${ }^{3}$, Jenifer D. Sánchez ${ }^{3}$, María A. Jones ${ }^{3}$
}

\section{Resumen}

Los procedimientos de diseño de canales a flujo libre y uniforme frecuentemente implican considerar coeficientes de la literatura, obviando el hecho que estos podrían diferir al cambiar la zona de estudio. El objetivo de este trabajo se centró en establecer el coeficiente de rugosidad de un canal, usando ecuaciones de flujo uniforme y compararlos con la literatura. Para ello, se usó un canal revestido transportando un flujo de $0.04 \mathrm{~m} 3 / \mathrm{s}$ con velocidades entre $0.33 \mathrm{~m} / \mathrm{s}$ y $0.357 \mathrm{~m} / \mathrm{s}$. El cálculo de los coeficientes se realizó considerando un tramo unidimensional de $10.5 \mathrm{~m}$ de longitud y $-0.4 \%$ en pendiente, efectuando mediciones cada $150 \mathrm{~cm}$. Los coeficientes obtenidos mediante las ecuaciones de Bazin y Manning presentan correlación muestral estable de +0.052 . El lecho estudiado presentó un coeficiente de rugosidad de Manning en promedio de 0.04 , siendo mayor a los valores asumidos en la literatura normalmente para canales nuevos.

Palabras clave: Canales abiertos, coeficiente de rugosidad de Manning, flujo uniforme, HEC-RAS, hidráulica de canales.

3 Facultad de Ingeniería y Arquitectura, Universidad Santo Tomás, Bucaramanga. Grupo de Investigación en Ingeniería Ambiental y Recursos Naturales - GINAM. nicolas.solanodustabuca.edu.co, https://orcid.org/0000-0001-8061-7518, monica.sandovaldustabuca.edu.co, https://orcid.org/0000-0001-7216-0407. Jennifer.sanchezQustabuca.edu.co, maria.jonesđustabuca.edu.co 


\section{Abstract}

The procedures for designing free and uniform flow channels frequently imply considering coefficients from the literature, ignoring that these could differ when changing the study area. This paper establishes the roughness coefficient of a channel, using steady flow equations and comparing them with the literature. For this, a lined channel transporting a flow of $0.04 \mathrm{~m}^{3} / \mathrm{s}$ with velocities between 0.33 and $0.357 \mathrm{~m} / \mathrm{s}$ was used. The coefficients were calculated considering a one-dimensional section of $10.5 \mathrm{~m}$ in length and $-0.4 \%$ in slope, making measurements every $150 \mathrm{~cm}$. The coefficients obtained using Bazin's equation and the Manning formula show a stable sample correlation of +0.052 . The studied bed had an average Manning's roughness coefficient of 0.04 , higher than the values usually assumed in the literature for new channels.

Keywords: Open channels, Manning's roughness coefficient, steady flow, HECRAS, channel hydraulics.

\section{Introducción}

En la actualidad se evidencia mayor intensidad en los cambios que se dan en la dinámica atmosférica (Nied, 2014). Lo anterior puede llegar a desencadenar, entre otras cosas, prolongados períodos de desabastecimiento de agua. Ante esto, se recurre al diseño de soluciones hidráulicas que, con frecuencia, abarcan un componente de incertidumbre cubierta por criterios que se fundamentan en suposiciones teóricas. Tal es el caso de los coeficientes de rugosidad de Manning (n) al momento de estimar la resistencia al flujo en diseños de canales hidráulicos a flujo uniforme.
Por lo anterior, se hace necesario que las estimaciones teóricas de resistencia al flujo se ajusten a las condiciones propias del problema que se quiere resolver, permitiendo un conocimiento del estado de los coeficientes de rugosidad de Manning presentes en cada canal, según sean las condiciones hidráulicas de funcionamiento para el flujo uniforme y las condiciones de uso y mantenimiento en las que se encuentre el canal.

Una estimación adecuada de las variables y parámetros que intervienen en los fenómenos hidráulicos resultará en períodos de vida útil más prolongados 
y optimización en tiempo y recursos. En normativas nacionales, el artículo 22 de la resolución No. 0330 de 2017 del Ministerio De Vivienda, Ciudad Y Territorio, fundamenta el ejercicio del diseño hidráulico, y establece que deberán ser incluidos esquemas, cálculos y modelaciones necesarias para la definición de obras, precisando parámetros tales como diámetros, caudales, velocidades, pendientes, especificaciones de materiales y demás aspectos técnicos que permitan asegurar el desempeño adecuado de los sistemas.

Diferentes estudios se han realizado para la estimación de los coeficientes de rugosidad de Manning en la hidráulica. Es así como Paria (1995) presentó un conjunto de tres fórmulas prácticas, con una base racional para el diseño de canales sin revestir. La familia de fórmulas propuesta por el autor fue obtenida mediante la combinación de funciones de resistencia al flujo, transporte de sedimentos y estabilidad de las márgenes del canal.

Otro estudio orientado a rugosidades presentes en los materiales con que se diseñan y construyen canales, Pantaleón et al., (2013) centraron su investigación en un revestimiento particular, en este caso el encachado. Los autores realizaron pruebas para diferentes valores de caudal, estimando los coeficientes de rugosidad de Manning en cada caso. De esta manera, concluyen, es necesario incorporar estimaciones de las condiciones de uso y mantenimiento del canal, a fin de obtener coeficientes para el diseño menos distantes de las condiciones de operación del canal. Los autores estimaron un valor de 0.025 en el coeficiente de rugosidad de Manning para el revestimiento estudiado.

Por otro lado, Li et al., (2000) indican que el coeficiente de rugosidad de Manning es uno de los parámetros más importantes para estudiar el flujo del agua sobre el suelo. Sin embargo, manifiestan que no está muy bien establecida una forma de determinar dicho parámetro de rugosidad en superficies de irrigación. Los autores presentan un método para determinar el coeficiente de rugosidad basado en un modelo analítico para superficies de irrigación, resuelto a través de iteraciones numéricas, con las cuales obtuvieron resultados consistentes de rugosidad considerando distintos tipos de revestimiento del canal.

Así mismo, Gutiérrez (2009) presenta en su estudio resultados obtenidos mediante pruebas de laboratorio, al evaluar el comportamiento del flujo en un 
canal hidráulico bajo distintos materiales de revestimiento tales como gravas, arenas y arcillas. Los valores que se obtuvieron para el coeficiente de rugosidad de Manning se mantuvieron cercanos a los expuestos en la literatura; a saber, 0.036 para gravas, entre 0.019 y 0.02 para arenas y entre 0.017 y 0.018 para arcillas. Adicionalmente, concluyen que pueden presentarse variaciones en dichos valores, producto de la erosión y la socavación en el canal, por lo cual, es importante relacionar estos factores a fin de obtener menos margen de error.

En este sentido, el objetivo de este trabajo fue conocer el estado del coeficiente de rugosidad de Manning a través de información primaria en un canal hidráulico revestido que conduce agua cruda, mediante fundamentos de flujo uniforme. De esta manera, se logró establecer el rango de velocidades las cuales, dependiendo de la capacidad de transporte simulada del canal, influyen en la resistencia al flujo, debido a fenómenos como desgaste y arrastre de material de revestimiento.

\section{Materiales y métodos}

El estudio se realizó en un canal hidráulico localizado en El Limonal perteneciente a la Universidad Santo Tomás, seccional Bucaramanga, el cual transporta agua cruda desde la parte alta del campus localizado en el municipio de Piedecuesta, parte baja de la Vereda El Granadillo, coordenadas $7^{\circ} 00^{\prime} 30.62^{\prime \prime} \mathrm{N}$ y $73^{\circ} 03^{\prime} 05.20^{\prime \prime} \mathrm{O}$.

La geometría hidráulica es de sección compuesta, determinada por una sección triangular en el fondo que se extiende $41.5 \mathrm{~cm}$ horizontalmente a cada lado desde el centro del canal, y una sección rectangular que se eleva verticalmente $52 \mathrm{~cm}$, incluyendo el borde libre. Esto genera un ancho promedio de 83 $\mathrm{cm}+/-1 \mathrm{~cm}$ en la sección de flujo uniforme en la que se desarrolló el estudio, y una profundidad máxima del canal de $52 \mathrm{~cm}$ en promedio.

Para determinar las condiciones hidrodinámicas del canal se identificó un tramo donde la condición de flujo predominante fuera uniforme, según definición, es decir, la observación de una lámina de agua estable e invariante en toda la longitud en dirección al flujo y para cualquier instante de tiempo (Moglen, 2015).

Con el fin de conocer las cargas dinámicas y de arrastre por descarga, se realizaron mediciones de caudal mediante aforos en un total de diez estaciones distanciadas $1.5 \mathrm{~m}$ cada una y en dirección 
del flujo aguas abajo. El equipo utilizado fue un micro molinete OTT (OTT HydroMet) con hélice No. 3, calibrado para procesar las velocidades de acuerdo con las revoluciones de la hélice en intervalos de 50 segundos. La técnica para los aforos se basó en el uso de dovelas, expresando la velocidad media para flujos permanentes y sin interrupciones como la registrada por el aforador de hélice medida al $60 \%$ desde la superficie libre al fondo.

Se realizó inspección visual del estado del revestimiento del canal, identificando antigüedad, tipo de mantenimiento y los aspectos más relevantes del lecho. La construcción lleva en operación 10 años, aproximadamente (hasta la fecha del presente trabajo). Se le realiza bajo mantenimiento. En cuanto al revestimiento utilizado, se encontró mortero de bajo mantenimiento con alta exposición de bolo de río de tamaño mediano y no superior a tres pulgadas de tamaño nominal.

Una vez identificado lo anterior, se tomó un tramo de condiciones de flujo estable para generar cálculos de diferentes capacidades de descarga simuladas, estimando, además, las velocidades que generan arrastre y desgaste en el revestimiento del canal.

\section{Resultados y discusión}

\section{Estimación de caudal y cálculo de los coeficientes de rugosidad}

La sección de estudio en el canal del campus El Limonal presentó una longitud de desarrollo de flujo uniforme en un tramo de $10.5 \mathrm{~m}$ en total, evaluado mediante secciones de dovelas individuales distanciadas a $1.5 \mathrm{~m}$, cada una. El tramo evaluado presentó una pendiente total de $4 \mathrm{~mm}$ por cada metro lineal, desplazando un flujo máximo de $0.04 \mathrm{~m}^{3} / \mathrm{s}$.

Los resultados de las dovelas individuales permiten apreciar el desarrollo de la condición de flujo uniforme en la cual las variaciones de perímetro mojado y radio hidráulico son imperceptibles, según se aprecia en la tabla 1.

Para estimar el factor de resistencia al flujo se utilizó la ecuación de flujo uniforme (ecuación 1), propuesta por Antoine Chèzy (como se citó en Chow, 1994), donde V es la velocidad media, $\mathrm{RH}$ es el radio hidráulico, sf es la pendiente del fondo y $\mathrm{C}$ es el factor de resistencia en cuestión, que se resolvió con la solución propuesta por Manning (ecuación 2) y Bazin (ecuación 3). Así mismo, con las soluciones propuestas 
por Manning y Bazin para dicho factor, expresó en términos de los coeficientes y las velocidades registradas mediante de rugosidad. el aforo realizado sobre las secciones, se

Tabla 1. Resumen de estaciones de la geometría hidráulica del canal estudiado.

\begin{tabular}{lllllll}
\hline Sección & $\begin{array}{r}\Delta X_{i} \\
(\mathrm{~m})\end{array}$ & $\begin{array}{r}\mathrm{Pi} \\
(\mathrm{m})\end{array}$ & $\begin{array}{r}\text { Rhi } \\
(\mathrm{m})\end{array}$ & $\begin{array}{r}\mathrm{Vi} \\
(\mathrm{m} / \mathrm{s})\end{array}$ & $\begin{array}{r}\text { Qi } \\
\left(\mathrm{m}^{3} / \mathrm{s}\right)\end{array}$ & $\begin{array}{r}\text { Qprom } \\
\left(\mathrm{m}^{3} / \mathrm{s}\right)\end{array}$ \\
\hline 8.0000 & 0.0000 & 1.0800 & 0.0963 & 0.3507 & 0.0365 & \\
7.0000 & 1.5000 & 1.0680 & 0.0993 & 0.3553 & 0.0377 & \\
6.0000 & 3.0000 & 1.0780 & 0.1030 & 0.3570 & 0.0396 & \\
5.0000 & 4.5000 & 1.0800 & 0.1037 & 0.3500 & 0.0392 & 0.0369 \\
4.0000 & 6.0000 & 1.0700 & 0.0953 & 0.3287 & 0.0335 & \\
3.0000 & 7.5000 & 1.0850 & 0.1010 & 0.3410 & 0.0374 & \\
2.0000 & 9.0000 & 1.0600 & 0.1015 & 0.3343 & 0.0360 & \\
1.0000 & 10.5000 & 1.0700 & 0.1028 & 0.3253 & 0.0358 & \\
\hline
\end{tabular}

Fuente: elaboración propia.

$$
\begin{aligned}
& V=C \sqrt{ } R H S_{f} \\
& C=\frac{1}{n} R H^{1 / 6} \\
& C=\frac{157.6}{1+\frac{m}{\sqrt{R H}}}
\end{aligned}
$$

Los cálculos permitieron identificar que el factor de resistencia al flujo del canal estudiado está determinado por un coeficiente de rugosidad de Manning en promedio de 0.0436. Esto evidencia -al compararse con los valores máximos para condiciones de operación similares propuestos por Chow (1994), los cuales no son superiores a 0.020 que es importante hacer un reajuste a las condiciones de operación del canal para prolongar su vida útil ya que actualmente las cargas dinámicas generan erosión, desgaste y arrastre de sedimento, producto del desprendimiento del revestimiento.

\section{Consistencia de los coeficientes}

Por otro lado, se encontró una adecuada consistencia en los cálculos obtenidos para el coeficiente de rugosidad de Manning al compararse con los coeficientes propuestos por Bazin, mediante las ecuaciones 2 y 3 , respectivamente, las cuales, como se mencionó anteriormente, expresan el factor de resistencia al flujo en función del coeficiente de rugosidad (n o m) y el radio hidráulico. La consistencia de los coeficientes de rugosidad adimen- 
sionales se presenta en la figura 1. Allí también se evidencia el modelo de ajuste lineal, el cual muestra una correlación de los coeficientes con un $\mathrm{R}^{2}$ de $96.4 \%$.

Por su parte, los coeficientes de variación para los coeficientes de Manning y Bazin fueron de 0.037 y 0.9859 , respectivamente. La tabla 2 presenta el resumen de los datos estadísticos como descriptores de tendencia central.
Tabla 2. Resumen de la información descriptiva de tendencia central de los datos.

\begin{tabular}{lcc}
\hline Descriptor de tendencia central & Bazin & Manning \\
\hline Promedio & 2.8663 & 0.0436 \\
Varianza & 7.9848 & 0.0000 \\
Desviación & 2.8257 & 0.0016 \\
Coeficiente de Variación & 0.9859 & 0.0370 \\
Correlación muestral & 0.0522 & \\
\hline
\end{tabular}

Fuente: elaboración propia.

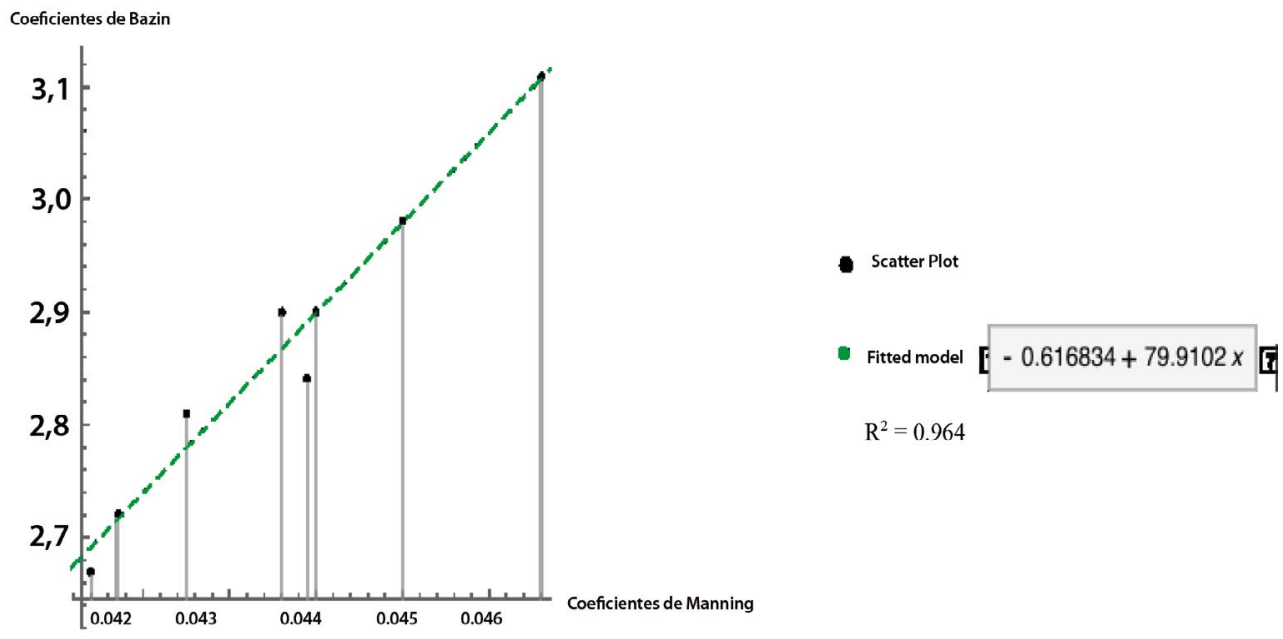

Figura 1. Correlación de los coeficientes de rugosidad de Manning y Bazin.

\section{Curva de descarga}

Una vez se conoció la consistencia y el comportamiento del flujo en términos de su resistencia a fluir, se calcularon los caudales que pueden presentarse en el canal a medida que se modifica el área mojada de su sección transversal median- te cambios en la profundidad, utilizando para ello el coeficiente de rugosidad de Manning promedio presentado en la tabla 2, y la ecuación de flujo uniforme (ecuación 1). La figura 2 presenta la curva de descarga obtenida, en la cual se puede apreciar que para una profundidad máxi- 
ma de $0.5 \mathrm{~m}$, la capacidad de descarga del rentes descargas que pueden presentarse canal es de $0.23 \mathrm{~m}^{3} / \mathrm{s}$, aproximadamente. en función de su profundidad. Es decir, se identifica en la curva las dife-

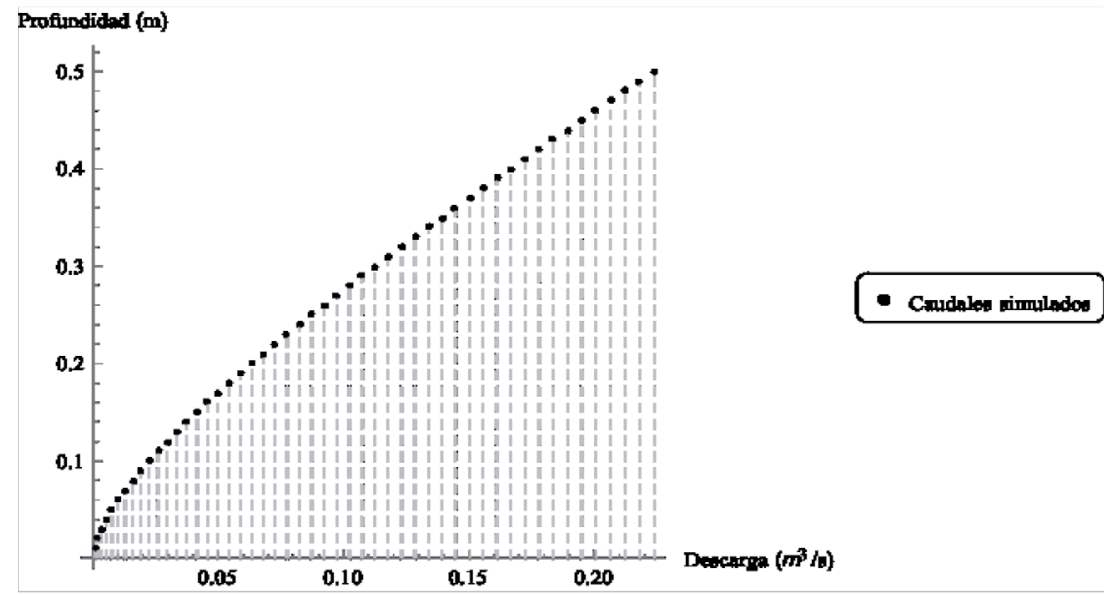

Figura 2. Curva de descarga obtenida para diferentes valores de profundidad. Fuente: elaboración propia.

\section{Curva de velocidades}

Con el fin de minimizar la erosión y desgaste del revestimiento por las cargas dinámicas que pueden presentarse a causa de velocidades elevadas, la figura 3 presenta la curva de velocidades que se obtiene para cada valor de caudal en función de la profundidad que se presente en el canal. Esta información es útil para mantener el caudal regulado de manera que no se generen incrementos superiores a las velocidades de operación. La curva de velocidades se com- porta de forma creciente a medida que la profundidad se incrementa, aunque estos en ningún momento superan los $0.6 \mathrm{~m} / \mathrm{s}$; concluyendo que hay una operación bajo condiciones subcríticas, teniendo en cuenta los caudales y las profundidades máximas que se pueden presentar en el canal.

Esta condición de régimen subcrítico con tendencia a estado crítico puede llegar a variar con la implementación de estructuras de ascenso y/o control en el canal, sin embargo, no se tuvieron en cuenta para el presente trabajo. 


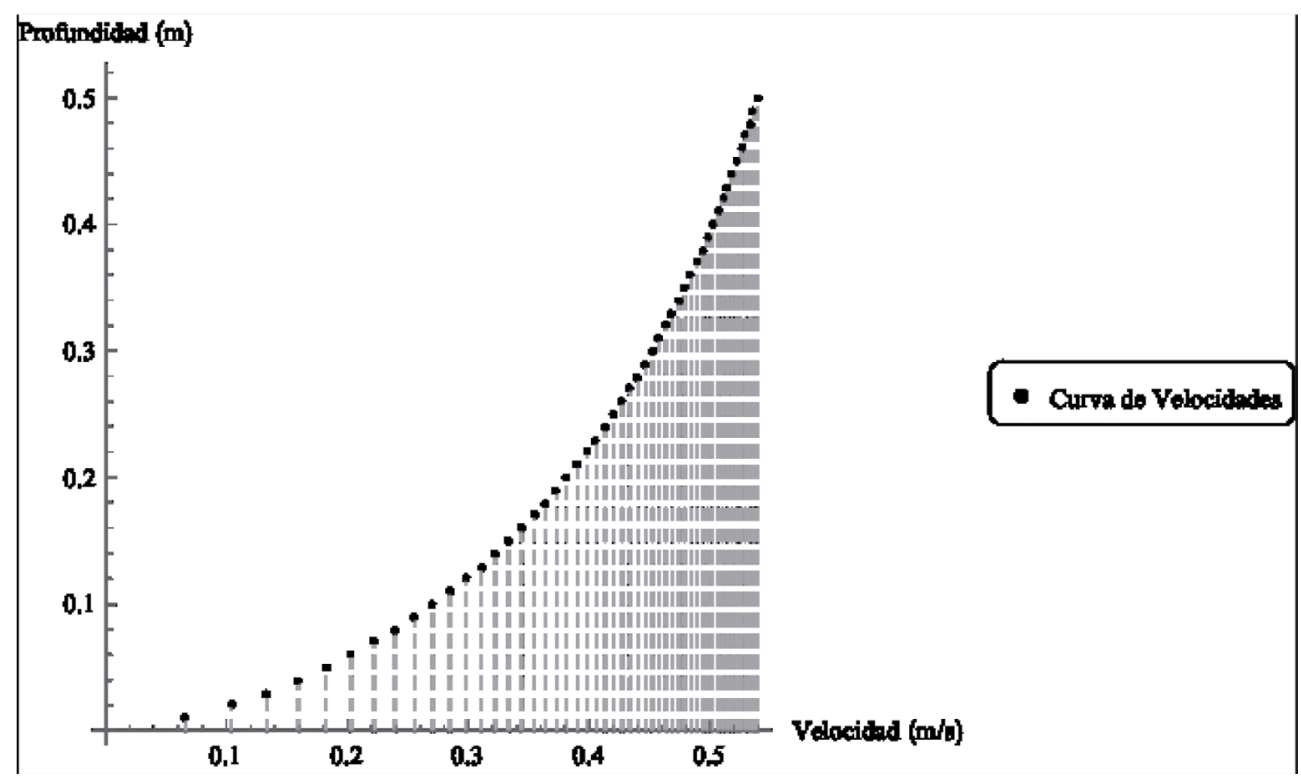

Figura 3. Curva de velocidades críticas en función de la profundidad.

Fuente: elaboración propia.

\section{Modelo HEC RAS}

Finalmente, se hizo el ajuste del modelo hidráulico en el software HEC RAS (River Analysis System), desarrollado por Centro de Ingeniería Hidrológica del Cuerpo de Ingenieros de la Armada de los Estados Unidos, estableciendo la resistencia al flujo mediante el coeficiente de rugosidad de Manning calculado para el revestimiento del canal. El comportamiento del modelo se mostró consistente para las condiciones de flujo estudiadas al momento de realizar las mediciones para el presente trabajo, las cuales fueron ingresadas al software. La figura 4 representa una de las estaciones aforada, en la cual se logran apreciar los niveles de profundidad crítica, nivel por debajo del cual el canal entraría en estado supercrítico a $7.5 \mathrm{~cm}$, aproximadamente, por debajo del nivel de borde libre. Así mismo, se presenta la línea del nivel de energía máximo alcanzado en el canal, de acuerdo con la representación de la sección tipo de la figura; su valor es de 12.5 $\mathrm{cm}$ medidos desde el fondo del canal.

Por otro lado, en la figura 5, se muestra el tramo de flujo uniforme modelado, en el cual se pueden apreciar las secciones de aforo que se realizaron para el desarrollo del modelo y del presente trabajo. 


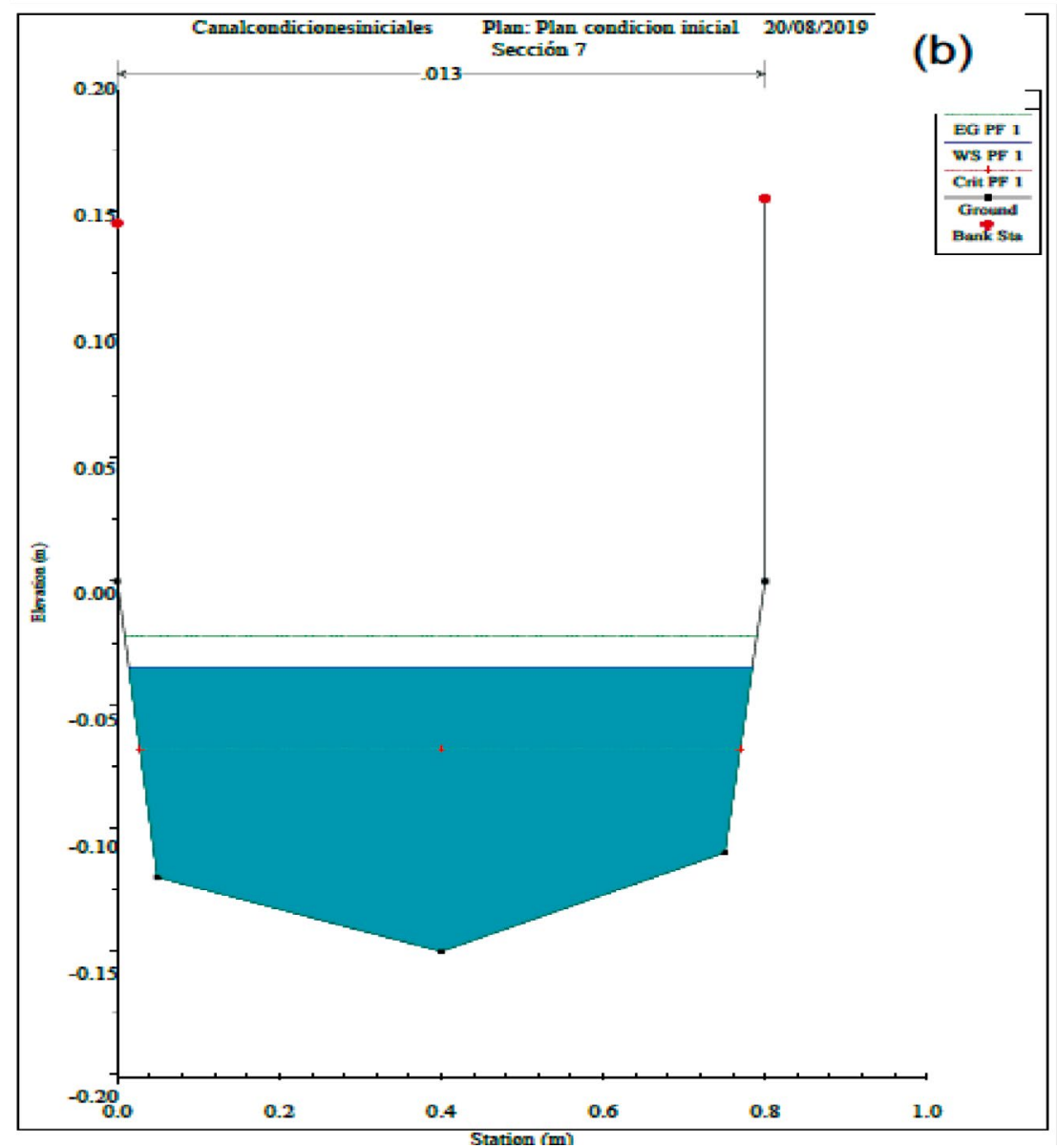

Figura 4. Estación de la sección transversal del canal en HEC - RAS.

Fuente: elaboración propia. 


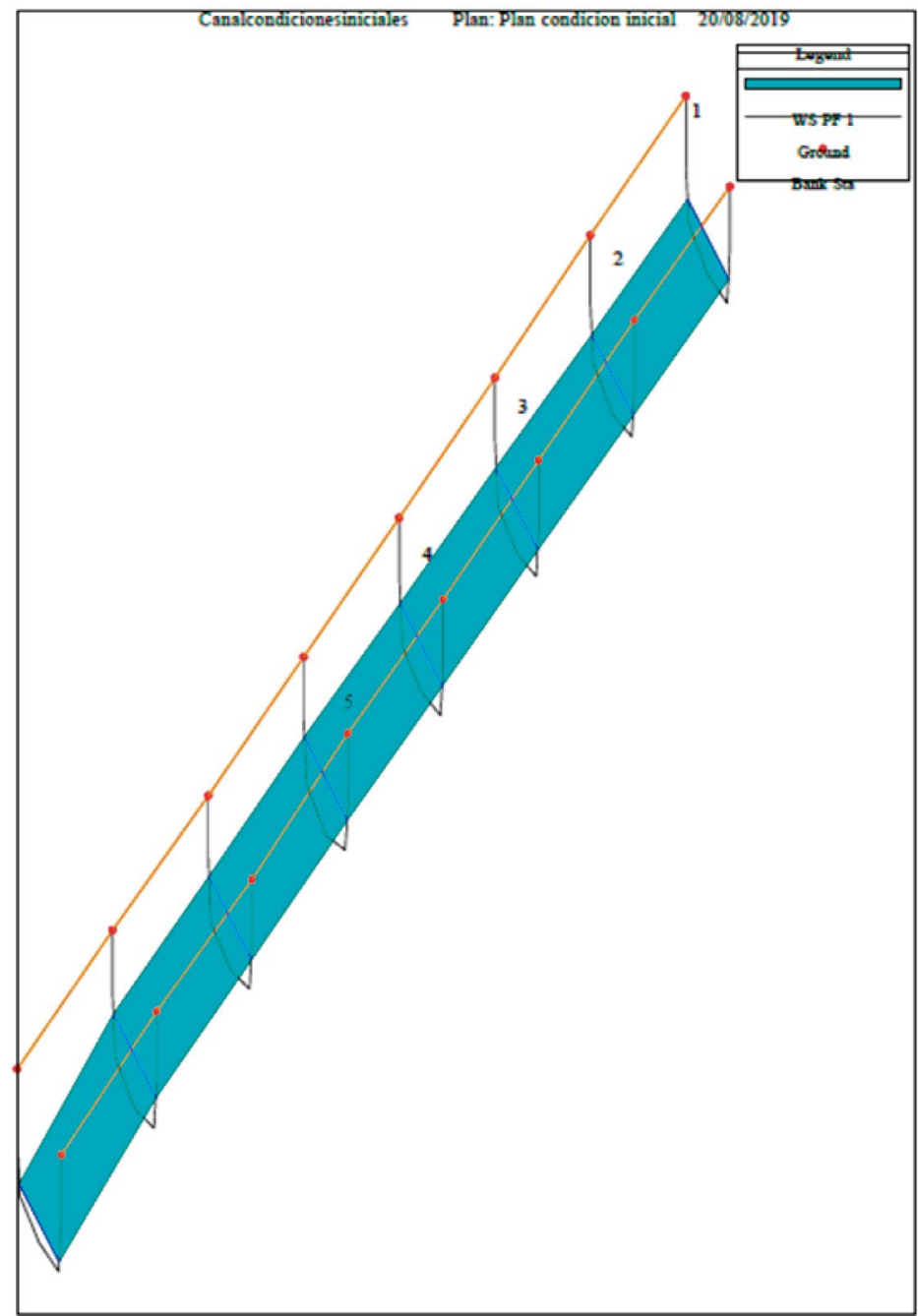

Figura 5. Lámina de agua del tramo de estudio en Flujo Uniforme con el software HEC - R Fuente: elaboración propia. 


\section{Conclusiones}

El presente trabajo determinó el coeficiente de rugosidad de Manning en un canal utilizado para transportar agua cruda en el campus agroindustrial de la Universidad Santo Tomás, seccional Bucaramanga. Los resultados mostraron que el tramo estudiado presenta un valor promedio de 0.0436 , el cual es elevado con respecto a los valores de diseño usados para canales nuevos con revestimientos similares (entre 0.013 y 0.02 ).

Las velocidades calculadas mostraron que el régimen se mantiene en condición subcrítica. Estos valores fueron obtenidos a partir de la curva de descarga para un rango de profundidades hasta $0.5 \mathrm{~m}$. Dicho régimen puede llegar a variar mediante estructuras de control o vertederos, sin embargo, esto no fue objeto de estudio en el trabajo.

El coeficiente de rugosidad de Manning que se obtuvo (0.0436) permitió evidenciar la necesidad de proporcionar una mejora al revestimiento del canal, en procura de prolongar su vida útil, minimizando la resistencia al flujo, la fricción, y la erosión de este por el transporte de agua cruda. La propuesta de mejora no se contempla dentro del alcance del trabajo presentado.

\section{Agradecimientos}

Los autores agradecen a la Universidad Santo Tomás, por la financiación realizada a través del proyecto de semilleros de investigación \#01948273.

\section{Referencias}

Chow, V. T. (1994). Hidráulica de canales abiertos. Bogotá, Colombia. McGraw-Hill.

Contreras, M. (1999). Apuntes de clase sobre flujo uniforme. Santiago de Chile, Chile. Universidad de Chile.

Gutiérrez, J. D. (2009). Cálculo del Coeficiente de Rugosidad de Manning utilizando gravilla, arena, piedra pegada y tierra como fondo mediante un canal a escala como modelo físico. Universidad de La Salle. Bogotá, Colombia.

Li, Z.; Zhang, J. (2000). Calculation of Field Manning's Roughness Coefficient. Agricultural Water Management 49 (2001) 153161. Beijing, China.

Ministerio De Desarrollo Económico, Dirección de Agua Potable (2000). Reglamento Técnico del sector de Agua Potable y Saneamiento Básico RAS - 2000. Bogotá, Colombia.

Ministerio De Vivienda, Ciudad Y Territorio. (2017). Resolución 0330 del 8 de junio de 2017. Bogotá, Colombia.

Moglen, G. E. (2015). Fundamentals of Open Channel Flow. CRC Press Taylor \& Francis Group. Florida. 
Nied, M et al. (2014). On the relationship between hydro-meteorological patterns and flood types. Journal of Hydrology 519 (2014) 3249-3262

Pantaleón, J.; Pérez, P.C.; Cordero, R.; Torres, A.; García, B. A.; Castillo, H. M.; Feliz, J. A.; Rivas, G. (2013). Determinación del coeficiente de rugosidad para encachado usado en el revestimiento de cunetas en la República Dominicana. Ciencia y Sociedad. Santo Domingo, República Dominicana.
Paria, H.D. (1995). Fórmulas prácticas para el diseño de canales sin revestir en terrenos aluviales. Ingeniería del Agua. Vol. 2 Núm. 3 (septiembre 1995) p. 53. Argentina.

Vatankhah, A. R. (2015). Analytical solution of gradually varied flow equation in circular channels using variable Manning coefficient. Flow Measurement and Instrumentation 43 (2015) 53-58. Tehran, Iran. 